\title{
The Effects of Contralateral Throwing Practice on Throwing Parameters, Isometric Strength, Grip Strength, and Shoulder Range of Motion in High School Baseball Athletes
}

\section{Raad Gheith ( $\nabla$ rgheith@theaspi.com )}

Applied Science and Performance Institute https://orcid.org/0000-0002-8444-4017

Matthew H. Sharp

Applied Science and Performance Institute

Matthew W. Stefan

Applied Science and Performance Institute

Dallen D. Reber

Applied Science and Performance Institute

\section{Salvatore LoDuca}

Robosport Technologies, LLC.

Ryan P. Lowery

Applied Science and Performance Institute

Jacob M. Wilson

Applied Science and Performance Institute

Research article

Keywords: Motor learning, contralateral training, cross-education, accuracy, velocity, strength

Posted Date: September 16th, 2020

DOI: https://doi.org/10.21203/rs.3.rs-61711/v1

License: (c) (1) This work is licensed under a Creative Commons Attribution 4.0 International License. Read Full License 


\section{Abstract \\ Background}

Baseball throwing is typically done with one dominant arm and after years of throwing the skills becomes autonomous and improvement is slowed. Incorporating training of the non-dominant arm could induce a cross-education effect and assist in improving the dominant throwing arm. Therefore, the purpose of this study was to determine the effect of contralateral (CL) throwing practice on dominant arm throwing accuracy and other parameters in experienced baseball players.

\section{Methods}

16 male high school baseball players participated in contralateral throwing practice. Each participant was randomly assigned to either contralateral $(\mathrm{CL})$ or control $(\mathrm{CON})$ following a pre-test. Sessions consisted of 30 non-dominant arm throws from a randomized location to a standard catch net, twice a week for 4 weeks. Following the 4 weeks of training, participants were post-tested to determine the effects of the contralateral throwing program. Accuracy percentage, throwing velocity, Accuracy:Velocity ratio, and nonspecific throwing parameters were collected to determine the effect of the contralateral throwing program.

\section{Results}

For dominant side throwing accuracy $\%$, significant pre- to post-testing differences were demonstrated in the $C L$ condition $(p<0.05$, Pre $=47.4$, Post $=54.9)$. Accuracy:Velocity ratio was statistically significant in the $C L$ condition in pre- to post-test $(p<0.05$, Pre $=0.71$, Post $=0.86)$. There were no significant differences detected in non-throwing specific parameters.

\section{Conclusion}

Contralateral throwing practice improved accuracy and Accuracy:Velocity ratio in the dominant arm of the $C L$ group. Use of a contralateral throwing program can be used to improve performance in a well learned, complex, open skill such as baseball throwing.

\section{Background}

Motor Learning is the relatively permanent increase in the capacity to express skill as a result of practice or experience (Bertram et al., 2017; Sawyer et al., 2001). Research indicates that learning occurs in at least three stages (Bertram et al., 2017). The first is known as the cognitive verbal stage in which learning is rapid and cognitive challenges and cortical output are at their greatest. These are followed by the associative and autonomous stages. During the associative stage, the learner has formed an internal 
representation of how to perform a given skill and refines it via auditory, and visual feedback. Learning is moderate during this phase as is cognitive effort. During the autonomous stage, cognitive effort and cortical output are at their lowest while the skill becomes automatic and learning is asymptotic or plateaued.

In baseball, throwing is typically done with one dominant arm. After years of practice this skill reaches an autonomous stage and improvement is slowed (Bertram et al. 2017). However, the untrained nondominant arm has room for rapid progress. Contralateral training $(C L)$ to induce a cross-education effect from a rapidly improving non-dominant arm may be a potential solution to elevate skill in the dominant arm. The cross-education of strength and skill learning was first discovered in 1894 by Scripture et al. (1894), who found that muscular strength and task steadiness (skill) could be improved in the contralateral limb following unilateral training. Cross-education of strength refers to the strength gain that is transferred to the contralateral limb following a unilateral training program in the ipsilateral limb. An extensive meta-analysis demonstrated an 18\% average increase in strength (Green et al., 2018A) via cross-education training. Indeed, extensive research has been done in strength training using very controlled unilateral movements. However, the improvement of motor skills is an important clinical aspect that has been widely overlooked in contralateral training literature (Green et al., 2018A). Recent research (Green et al., 2018B) has demonstrated that training in the contralateral arm reduced variability of force in the ipsilateral arm indicating an increase in skill. To date, however, we lack research in more complex and open skills such as the throwing that occurs in baseball.

Indirect research demonstrates that baseball players who bat opposite of their dominant throwing arm (e.g. left-handed hitter, right-handed thrower) demonstrate greater baseball performance than those who bat and throw on the same side (Mann et al., 2017). These data indicate that contralateral training may open pathways in the brain that are essential to baseball performance. Moreover, our recent data demonstrated that the combination of randomized contralateral batting practice can improve performance in the dominant swing (Sharp et al., 2020). However, the cross-education effect in welltrained baseball players who have plateaued, or reached the autonomous stage in throwing accuracy, has yet to be examined.

The primary purpose of this study was to examine the effects of contralateral throwing on accuracy and the accuracy to velocity ratio in the dominant limb during a season in well-trained baseball players. The second purpose was to examine if cross-education generalizes to other related but not specific parameters to baseball such as range of motion, strength, and other kinetic variables. We hypothesized that contralateral throwing meant to improve accuracy will improve accuracy in the dominant arm. We also hypothesized improvement in non-general parameters, albeit to a lesser extent.

\section{Methods}

\section{Subjects}


A total of 16 healthy high school baseball players with at least 6 years' experience of organized baseball play comprised the subject pool. Subjects mean age was 16 years old ( $S D=0.9$ years), mean height of $179.9 \mathrm{~cm}(\mathrm{SD}=6.0 \mathrm{~cm})$, and mean weight of $76.8 \mathrm{~kg}(\mathrm{SD}=15.8 \mathrm{~kg})$. The acceptable experience level for subjects was at least six consecutive years of organized baseball competition. The subjects needed to be free of musculoskeletal injuries within the previous six months at the start of the study. All subjects were right-handed and right-eye dominant. Eligibility was determined during screening prior to enrollment.

\section{Familiarization}

Subjects underwent two familiarization sessions separated by 48 hours to be acquainted with testing procedures. Both familiarization sessions were completed at least one week prior to the commencement of the study.

\section{Training Protocol}

All subjects continued their regular practice schedules during practice using the dominant arm. However, the experimental group also included 30 contralateral throws performed 3 days per week for 4 weeks. For each training session, subjects stood with their backs against the target (catch net), retrieved a baseball from the ground placed at one of three locations; turned, stepped and threw into a catch net. The catch net remained in a stagnant location for all training sessions. Subjects were instructed to release the ball behind a throwing line marked $50 \mathrm{ft}$ from the catch net. The catch net (PitchersPocket9, BetterBaseball, Marietta, GA) measures is 36in wide, 42in tall, and 48in diagonal including the framed cushion. Within the frame of the catch net was 9 catch pockets measuring approximately 10 in wide by 12 in tall. Subjects were being instructed to aim for the center most catch pocket for every throw (Fig. 1). After each throw, subjects reset with their back to the catch net and repeat the procedure for 30 throws. An illustration of the training procedure is provided in Fig. 2.

\section{Measurements}

\section{Throwing Performance}

To assess throwing performance objectives, all players performed a standardized throwing performance test at pre- and post-testing. The test included 30 pitches in total that were all thrown with the dominant arm and non-dominant arms. Subjects stood with their backs against the target (catch net), retrieved a baseball from the ground; turned, stepped and threw into the catch net. Subjects were instructed to release the ball behind a throwing line marked 50ft from the catch net and aim for the center most catch pocket for each throw. The location of the catch net and baseball remained constant for pre- and posttesting for each of the 30 throws (Fig. 3). All throws were scored according the to the following: center pocket $=5$ points, surrounding pockets $=3$ points, foam padding $=1$ point, miss $/$ other $=0$ points (Fig. 4 ). A radar gun will be place behind the area of ball retrieval to velocity of each throw. This was used for measurement of the Accuracy:Velocity ratio. 
Table 1

Dependent Variables for Throwing Performance.

\begin{tabular}{|c|c|}
\hline Variable & Description \\
\hline $\begin{array}{l}\text { Throwing } \\
\text { Velocity (V) }\end{array}$ & Velocity of a given pitch in miles per hour (mph). \\
\hline $\begin{array}{l}\text { Maximum Pitch } \\
\text { Speed }\left(P V_{\max }\right)\end{array}$ & $\begin{array}{l}\text { Maximum velocity of a pitch in miles per hour (mph). } \mathrm{PV}_{\max } \text { for a target hit and } \\
\text { target miss for each assigned target will be recorded. }\end{array}$ \\
\hline $\begin{array}{l}\text { Minimum Pitch } \\
\text { Speed }\left(P V_{\min }\right)\end{array}$ & $\begin{array}{l}\text { Minimum velocity of a pitch in miles per hour (mph). } \mathrm{PV}_{\min } \text { for a target hit and } \\
\text { target miss for each assigned target will be recorded. }\end{array}$ \\
\hline Target Hits & Tally of correct pitch placement. \\
\hline Target Misses & Tally of incorrect pitch placement. \\
\hline Accuracy \% & $\begin{array}{l}\text { Total points achieved divided by } 150 \text { (maximum value achievable), multiplied by } \\
100 .\end{array}$ \\
\hline $\begin{array}{l}\text { Accuracy-to- } \\
\text { Velocity Ratio }\end{array}$ & Accuracy $\%$ divided by the average velocity (a.u.) \\
\hline
\end{tabular}

\section{Hand Grip Strength}

Maximal hand grip strength was assessed in the dominant and non-dominant hand via hand grip dynamometry. Subjects were asked to stand with the dynamometer in hand with the arm parallel to the body without squeezing the arm against the body. The width of the handle was adjusted to the size of the hand such that the middle phalanx rested on the inner handle. Subjects were allowed to perform one testing trial. Thereafter, subjects underwent three testing trials separated by 2 minutes rest. The best result of the three testing trials was recorded in kilograms and used for analysis. Subjects were asked to exert maximal effort and verbal encouragement will be used for the testing trials. Hand grip strength was assessed at pre- and post-testing.

\section{Shoulder Isometric Dynamometry}

Maximal voluntary isometric contraction (MVIC) of internal and external shoulder rotation was assessed on the dominant arm using a dynamometer (Biodex System 3, Biodex Medical System; New York, USA). Prior to testing, the dynamometer will be calibrated to the manufacturer's guidelines. Internal and external range of motion values were collected upon calibration prior to testing. Subjects were seated at the respect to the dynamometer with the shoulder in $90^{\circ}$ of abduction, the elbow in $90^{\circ}$ of flexion, and the forearm/wrist neutral. Stabilization belts included Velcro fastened across the trunk, pelvis, and thigh to prevent movement compensation. The testing protocol consisted of 3 sets of 3 repetitions of MVIC of internal and external shoulder rotation in an alternating fashion separated by 60 seconds rest (i.e. internal rotation, $60 \mathrm{~s}$ rest, external rotation, $60 \mathrm{~s}$ rest, etc.). Each maximal isometric contraction last 5 seconds. Strong verbal encouragement was used during the testing bout. The data recorded for analysis was the 
highest peak torque achieved in the 3 sets for each leg expressed in absolute (ft-lbs.) and relative (ftlbs. $/ \mathrm{kg}^{-1}$ ) values. Shoulder dynamometry testing occurred at pre- and post-testing.

\section{Statistical Analysis}

Prior to performing inferential statistics, normality of the data was confirmed with Shapiro-Wilk test ( $p$ > 0.05 ) and no significant between group differences was observed for any dependent variables at pretesting $(p>0.05)$. Two-way repeated measures analysis of variance (ANOVA) was performed assuming group and time as fixed factors. Whenever a significant F-value was obtained, a post-hoc test with Tukey's adjustment was performed for multiple comparison purposes. Whenever p-values for the Ftest indicated a trend towards significance, the absolute mean change from pre- to post-testing was calculated for each subject and unpaired t-test were used to compare conditions. All statistical analysis was performed using GraphPad Prism (Version 7, San Diego, CA, USA). The alpha level was set a $p \leq$ 0.05. Data are reported as mean \pm standard error (SE).

\section{Results}

\section{Throwing Parameters}

Pre to post values for dominant throwing accuracy in the $\mathrm{CL}$ condition had an absolute mean (delta) increase if 7.5, an increase that was $5 x$ greater than the CON condition. The increase in the CL condition was significant $(p \leq 0.05)$ whereas the increase in the CON was not. Pre to Post values for Non-Dominant Throwing Accuracy in the CL condition demonstrated a percent increase of $40.2 \%$ whereas the CON condition did not change. This increase in the CL condition was statistically significant $(p \leq 0.05)$ (Fig. 1a).

There were no detectable changes in either condition for dominant side average throwing velocity during the retention test (Fig. 1b). Pre to post values for the dominant side Accuracy:Velocity ratio in the CL condition demonstrated a percent increase of $18.2 \%$, a statistically significant increase $(p<0.05)$. No significant differences were demonstrated by the $\mathrm{CON}$ condition (Fig. 1c). This variable is quantified as (Accuracy \%/Velocity) and expressed in arbitrary units.

\section{Non-Throwing Specific Parameters}

There was a significant difference in non-dominant grip strength in the CL condition $(p<0.05)$. There were no significant differences in dominant grip strength. No significant differences were detected in either condition for internal rotation peak torque, external rotation peak torque, and total arc internal/external range of motion (Table 1). 
Table 1

Non-Throwing Specific Parameters.

\begin{tabular}{|lllll|}
\hline \multicolumn{4}{l}{ CL } & \multicolumn{3}{ll}{ CON } & \\
\hline Variable & DOM & ND & DOM & ND \\
\hline Grip Strength (kg) & & & & \\
\hline Pre & $49.3(2.8)$ & $42.1(3.4)$ & $45.1(2.8)$ & $44.9(2.8)$ \\
\hline Post & $50.4(2.9)$ & $47.2(3.3)^{\star}$ & $43.4(2.6)$ & $45.7(3.5)$ \\
\hline Int. Rot. Peak Torque (ft-lbs.) & & & & \\
\hline Pre & $37.9(4.1)$ & $29.4(4.2)$ & $34.0(2.9)$ & $34.6(4.6)$ \\
\hline Post & $37.5(3.2)$ & $31.2(3.6)$ & $34.7(3.3)$ & $34.7(4.1)$ \\
\hline Ext. Rot. Peak Torque (ft-lbs.) & & & & \\
\hline Pre & $22.7(1.7)$ & $19.0(1.9)$ & $18.4(1.4)$ & $21.6(1.8)$ \\
\hline Post & $22.7(1.4)$ & $17.2(1.3)$ & $18.3(1.5)$ & $21.1(1.7)$ \\
\hline Int Shoulder Rot. ROM (deg) & & & & \\
\hline Pre & $42.9(3.6)$ & $51.6(4.3)$ & $44.0(3.5)$ & $56.7(5.7)$ \\
\hline Post & $41.1(3.2)$ & $49.9(4.1)$ & $46.5(3.8)$ & $50.2(4.4)$ \\
\hline Ext Shoulder Rot. ROM (deg) & & & & $89.4(5.7)$ \\
\hline Pre & $98.1(5.6)$ & $92.1(6.3)$ & $103.3(6.5)$ & $89.8)$ \\
\hline Post & $98.2(5.2)$ & $90.4(6.1)$ & $101.4(6.8)$ & $88.4(5.4)$ \\
\hline Results are expressed as Mean (SE). & & & \\
\hline *Significantly different than Pre (p<0.05) & & & \\
\hline
\end{tabular}

\section{Discussion}

The primary purpose of this study was to examine the effects of contralateral throwing practice in the non-dominant arm on accuracy and the Accuracy:Velocity Ratio in the dominant limb during a season in well-trained baseball players. The second purpose was to examine if cross-education generalizes to other related but not specific parameters to baseball such as range of motion, strength, and other kinetic variables. We hypothesized that contralateral throwing meant to improve accuracy would improve accuracy in the dominant arm. We also hypothesized improvement in non-general parameters. Albeit, to a lesser extent. The primary hypothesis was supported in that dominant arm accuracy increased by $19 \%$ and the Accuracy:Velocity Ratio by $18.2 \%$. The second hypothesis was generally not supported. We found 
that improvements did not occur in any parameters except in grip strength in the non-dominant arm and that this did not transfer to the dominant arm.

The overwhelming majority of research in cross-education literature has taken place in general strength parameters. A meta-analysis of research demonstrated an $18 \%$ average increase in strength in the nontrained arm when exercising in a contralateral manner (Green et al., 2018 A). However, the improvement of motor skills is an important clinical aspect that has been widely overlooked in contralateral training literature. Our research was the first that we are aware of that investigated the cross-education effects of a highly complex sports specific skill such as throwing accuracy. We found strong agreement with strength literature demonstrating a $19 \%$ increase in throwing accuracy in an otherwise asymptotic group of athletes as demonstrated in the control condition. The $40 \%$ increase in non-dominate throwing performance indicates that these athletes were likely 'thrown' back into the non-verbal rapid learning phase of Fitts and Posner's learning model. It is in this stage where theoretical cortical activation is at its greatest and performance improvements are substantial as learning increases (Bertram et al., 2017; Sawyer et al. 2000).

We posited that underlying measurable physiological parameters such as improved torque and range of motion and/or general strength might underlie changes in performance. However, this generalized hypothesis was not supported. While we did not examine other explanations, scientists have proposed two major frameworks to explain cross-education effects (Ruddy et al., 2013). The first include "crossactivation models." These models are centered on the observation that contralateral movements result in bilateral increases in corticospinal excitability (Ruddy et al., 2013). The associated speculation is that such generalized activity, when present during contralateral practice, leads to concurrent adaptations in neural circuits that project to the untrained arm; thus, improving in this case throwing accuracy. Alternatively, "bilateral access" models entail that motor programs formed during contralateral practice, may subsequently be utilized on the other limb-that is, by the motor program that constitutes the control centers for movements of both limbs (Park et al., 2003). Considering that cortical activity is greatest during the cognitive verbal phase of learning, it is possible that greater activation improved performance on the dominant arm. However, to date, this is speculative in our throwing experiment and remains to be studied.

With the exception of strength, we did not see a generalized increase in nonspecific parameters in the non-dominant arm that was trained in a contralateral manner. As such, transfer would not be expected in those parameters. These results agreed with past literature which suggested that transfer of skills and strength are task specific (Behm et al., 1993; Bachman et al., 1961). Moreover, research suggests that in less complex and simple tasks like grip strength that neural adaptations are plateaued at an early phase of learning (the first few months) (Gabriel et al., 2006). Thus, it may be that transfer of the non-dominantto-dominant arms did not occur due to maxed out neural adaptations in the dominant arm.

In baseball throwing activities, it has been well described and demonstrated that accuracy is greater at lower velocities (Freeston et al., 2015). In the current study, we observed an improvement in accuracy 
without a sacrifice in velocity following contralateral throwing practice. This was indicated by an improvement in the ratio of accuracy to velocity (Fig. 4). Indeed, since the time of Fitts (see Kovacs et al., 2008 for explanation) it has been known that there is a strong inverse relationship between velocity and accuracy. Researchers believe that a hallmark of expertise is the ability to sustain high velocities while demonstrating greater than average or excellent improvements in accuracy (Englehorn, 1997). While we are uncertain of why this ratio improved, previous research in strength literature has shown a decrease in force variation that transferred from contralateral training to the untrained limb (Green et al. 2018A). Future research will need to investigate if this underlies the changes we see in this study.

One reason to potentially account for the lack of changes in non-throwing specific parameters such as range of motion and grip strength is task specificity in training. The primary focus of the training was accuracy; therefore, we would expect improvements in throwing accuracy but not velocity or non-specific throwing parameters. Hubbard et al. (2009) stated that increases in learning are task specific as learning is maximal in the task that is being trained. If the training regimen focused on improving velocity over the 4 weeks then we would expect to see velocity and potentially strength improvements as training effects are confined to the practiced task (Schubert et al., 2008). Including a strength training protocol and maximal intent throws to enforce strength and velocity in addition to accuracy training could be a solution to improving all aspects of baseball throwing. However, future research will need to investigate the impact of contralateral strength and accuracy training on complex and open skills such as baseball throwing.

Although this study did not focus in this area, future research should investigate the efficacy of a contralateral randomized training in rehabilitation and injury prevention (i.e. prehab). Such a training program could assist athletes returning from injury to minimize the losses in strength and accuracy due to inactivity, or aid in prevention of injury as performance in the dominant limb, which is commonly overused, could improve through training of the neglected, non-dominant limb

\section{Conclusion}

In conclusion, we demonstrate for the first time that contralateral training of the non-dominant arm in well trained baseball players robustly improves throwing accuracy and the Accuracy:Velocity ratio of the dominant throwing arm. These findings are important as skilled athletes become asymptotic in their performances as their training age increases. Contralateral training, even in fairly advanced athletes, may provide a viable solution to this eminent problem.

\section{Abbreviations}

CL: contralateral; CON:control; Int:Internal; Ext:External; deg:degress; ft-lbs.:foot pounds; ANOVA:analysis of variance

\section{Declarations}




\section{Ethics Approval and Consent to Participate}

Prior to the commencement of the study, written informed consent was obtained from the subjects and their parent(s) or legal guardian(s). The procedures carried out in this study were approved by an Institutional Review Board (IntegReview, Austin, TX; Protocol \#051902).

\section{Consent for publication}

Not applicable.

\section{Availability of data and materials}

The datasets during and/or analyzed during the current study available from the corresponding author on reasonable request.

\section{Competing Interests}

Salvatore LoDuca is the Owner and CEO of RoboSports Technologies, LLC. However, he was not involved in carrying out the investigation, data curation, or formal data analysis. Other authors declare no other relevant affiliations or competing interests with the subject matter or materials in this manuscript.

\section{Funding}

This study was funded by RoboSports Technologies, LLC.

\section{Author Contributions}

Conceptualization, J.M.W, S.L and M.H.S; data curation, M.H.S, R.H.G, M.W.S, and D.D.R; formal analysis, M.H.S; investigation, M.H.S, R.H.G, M.W.S, D.D.R, and R.P.L; methodology, J.M.W, M.H.S, R.H.G, M.W.S, D.D.R, and R.P.L, supervision, J.M.W and M.H.S; writing- original draft, J.M.W, M.H.S, and R.H.G; writingreviewing and editing, J.M.W, M.H.S, R.H.G, and M.W.S. All authors have read and agreed to the published version of the manuscript.

\section{References}

1. Bachman JC. Specificity vs. generality in learning and performing two large muscle motor tasks. Research Quarterly American Association for Health Physical Education Recreation. 1961;32(1):311.

2. Behm DG, Sale DG. Velocity specificity of resistance training. Sports Med. 1993;15(6):374-88.

3. Bertram CP, Guadagnoli MA, Marteniuk RG. (2017). THE STAGES OF LEARNING AND IMPLICATIONS FOR OPTIMIZED LEARNING ENVIRONMENTS. Routledge International Handbook of Golf Science. 
4. Engelhorn R. Speed and accuracy in the learning of a complex motor skill. Percept Mot Skills. 1997;85(3):1011-7.

5. Freeston J, Ferdinands RE, Rooney K. The Launch Window Hypothesis and the Speed-Accuracy Trade-Off in Baseball Throwing. Percept Mot Skills. 2015;121(1):135-48.

6. Gabriel DA, Kamen G, Frost G. Neural adaptations to resistive exercise. Sports Med. 2006;36(2):13349.

7. Green LA, Gabriel DA. The cross-education of strength and skill following unilateral strength training in the upper and lower limbs. J Neurophysiol. 2018A;120(2):468-79.

8. Green LA, Gabriel DA. The effect of unilateral training on contralateral limb strength in young, older, and patient populations: a meta-analysis of cross-education. Physical Therapy Reviews. 2018B;23(4-5):238-49.

9. Hubbard IJ, Parsons MW, Neilson C, Carey LM. Task-specific training: evidence for and translation to clinical practice. Occupational therapy international. 2009;16(3-4):175-89.

10. Kovacs AJ, Buchanan JJ, Shea CH. Perceptual influences on Fitts' law. Exp Brain Res. 2008;190(1):99-103.

11. Mann DL, Loffing F, Allen PM. The Success of Sinister Right-handers in Baseball. N Engl J Med. 2017;377(17):1688-90.

12. Park JH, Shea $\mathrm{CH}$. Effect of practice on effector independence. Journal of motor behavior. 2003;35(1):33-40.

13. Schubert M, Beck S, Taube W, Amtage F, Faist M, Gruber M. Balance training and ballistic strength training are associated with task-specific corticospinal adaptations. Eur J Neurosci. 2008;27(8):2007-18.

14. Scripture EW, Smith n, Brown EM. On the education of muscular control and power. Stud Yale Psychol Lab 1894; 2:114-9.

15. Sharp MH, Gheith RH, Reber DA, Stefan MW, Loduca S, Lowery RP, Wilson JM. The effect of blocked versus random practice on dominant and non-dominant baseball swing. Journal of Sport Human Performance. 2020;8(1):1-9. DOl:https://doi.org/10.12922/jshp.v8i1.

\section{Figures}




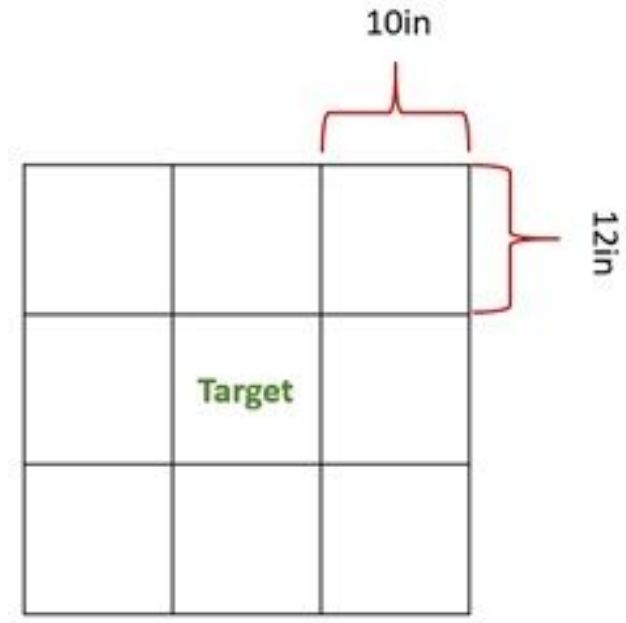

Figure 1

Illustration of the catch net.

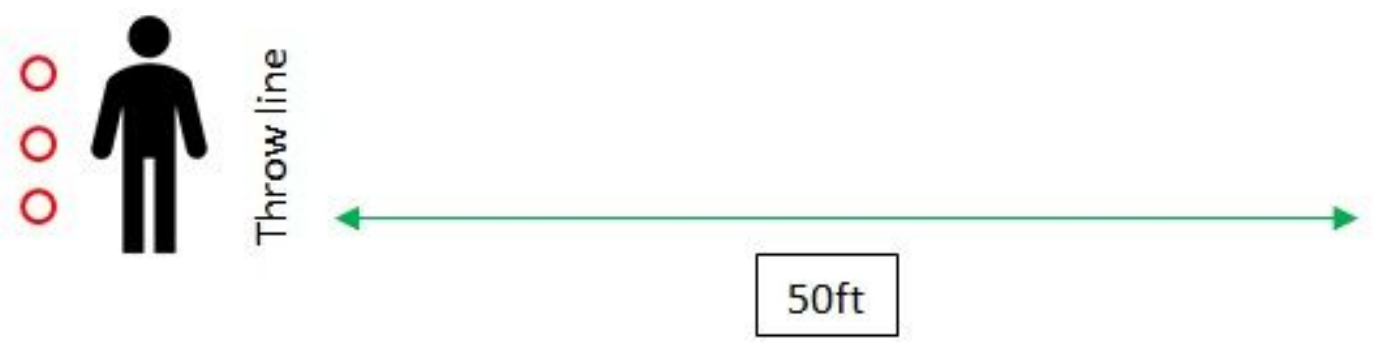

Catch

Net

Figure 2

Illustration of training procedure.
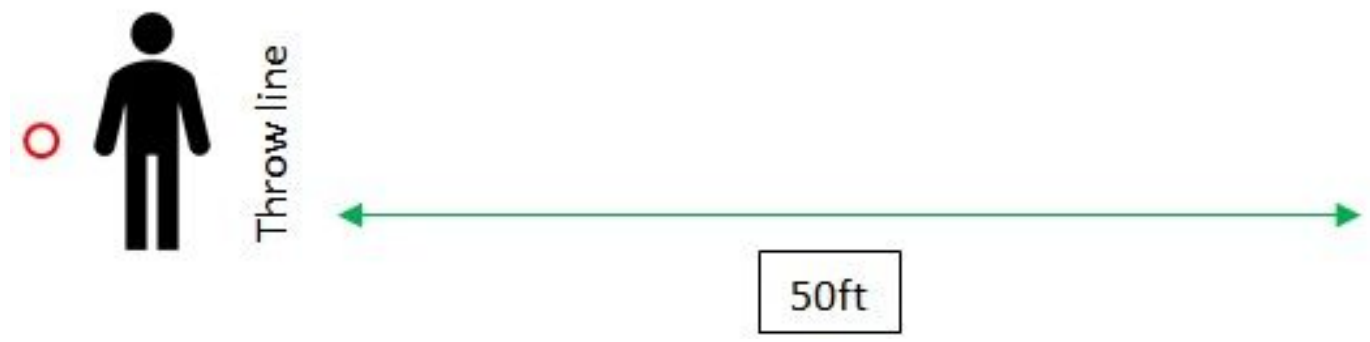

\section{Catch \\ Net}

Radar

Gun

Figure 3

Illustration of pre-and post-testing procedure. 


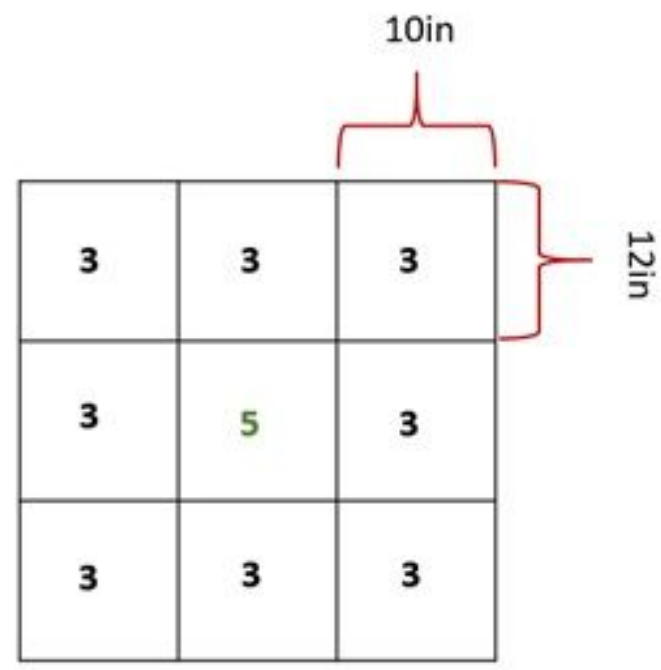

Figure 4

Illustration of the catch net scoring system.

A

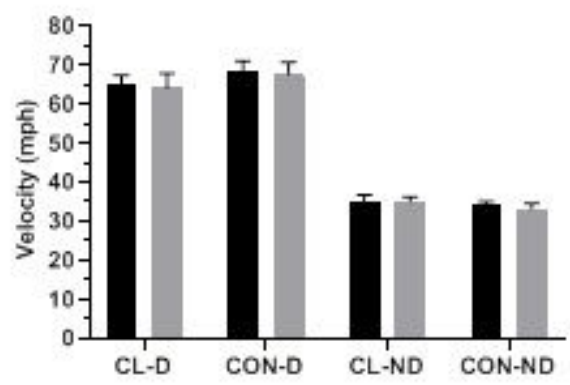

B

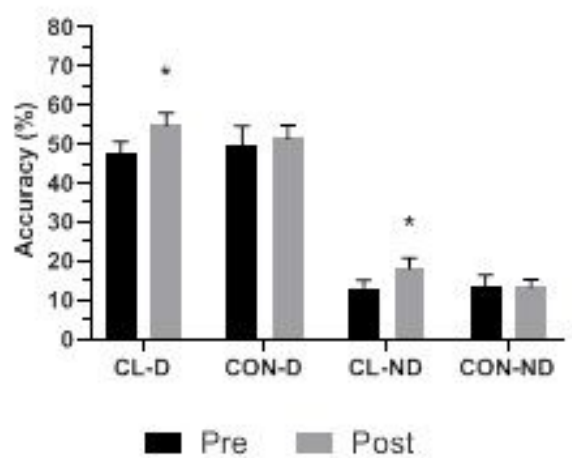

C

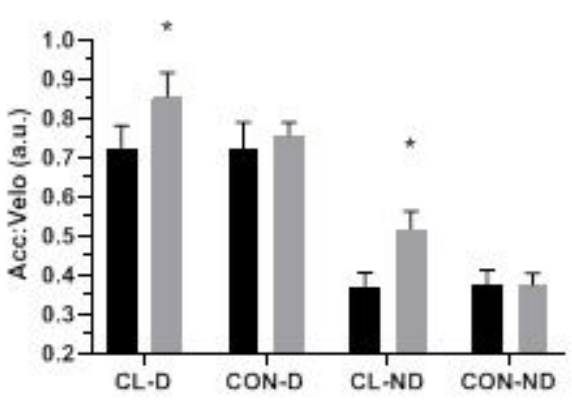

Figure 5

Pre- and Post- Throwing Velocity (A), Accuracy (B), and Accuracy:Velocity (C) on Dominant and NonDominant Throwing Arm. 